\title{
Bacterial lipopolysaccharide recognition by surfactant protein D
}

\author{
A.K. Shrive ${ }^{1}$, J.R. Littlejohn ${ }^{1,2}$, H.M. Williams ${ }^{1,3}$, W. Neale ${ }^{1}$, S. Collister ${ }^{1}$, D. Hood ${ }^{4}$, S. Oscarson ${ }^{5}$, J. Madsen ${ }^{6}$, H.W. \\ Clark $^{6}$, T.J. Greenhough ${ }^{1}$ \\ ${ }^{1}$ School of Life Sciences, Keele University, U.K., \\ ${ }^{2}$ Current address: School of Biochemistry, University of Bristol, U.K., \\ ${ }^{3}$ Current address: Bernhard Nocht Institute for Tropical Medicine, Department of Virology, Hamburg, \\ ${ }^{4}$ Mammalian Genetics Unit, MRC Harwell Institute, Harwell Science and Innovation Campus, U.K., \\ ${ }^{5}$ School of Chemistry, University College Dublin, Ireland, \\ ${ }^{6}$ EGA Institute for Women's Health, Faculty of Population Health Sciences, University College London, U.K.
}

a.k.shrive@keele.ac.uk

Human surfactant protein D is a collectin and member of the C-type lectin superfamily of proteins that forms an essential part of the mammalian innate immune system. The collectins have been recognised to not only bind to invading pathogens, allowing for recognition by immune cells, but also play an important role in activating and regulating the response of both the innate and acquired immune systems. Crystal structures of a biologically and therapeutically active recombinant homotrimeric fragment of human SP-D (hSP-D) complexed with the inner core oligosaccharides from gram negative bacterial human pathogens Haemophilus influenzae, Salmonella enterica sv Minnesota rough strains [1-2] and Escherichia coli provide unique multiple insights into the recognition and binding of bacterial lipopolysaccharide (LPS) by hSP-D.

The structures reveal that hSP-D specifically and preferentially targets the LPS inner core via calcium dependent recognition of the proximal inner core heptose dihydroxyethyl side chain coupled with specific interactions with the binding site flanking residues Arg343 and Asp325 and evidence for an extended binding site for LPS inner cores. Where this preferred mode of binding is precluded by the crystal lattice, the structures show that hSP-D has the flexibility and versatility to adopt alternative strategies for bacterial recognition, utilising alternative LPS epitopes including terminal or non-terminal sugars. Alongside binding studies of both whole bacteria and LPS the structures also demonstrate that carbohydrate extensions to the core LPS oligosaccharide, previously thought to be targets for collectins, are important in shielding the more vulnerable target sites in the LPS core. Recent structures of hSP-D bound with small synthetic LPS core component ligands which include phosphorylation of specific carbohydrate residues further demonstrate not only the ability to adopt alternative modes of recognition but also that LPS phosphorylation may provide an additional mechanism by which pathogens can efficiently evade a first-line mucosal innate immune defence.

[1] Clark, H. W., Mackay, R. M., Deadman, M. E., Hood, D. W., Madsen, J., Moxon, E. R., Townsend, J. P., Reid, K. B. M., Ahmed, A., Shaw, A. J., Greenhough, T. J. \& Shrive, A. K. (2016). Infection \& Immunity 84, 1585.

[2] Littlejohn, J. R., da Silva, R. F., Neale, W. A., Smallcombe, C. C., Clark, H. W., Mackay, R. M. A., Watson, A. S., Madsen, J., Hood, D. W., Burns, I., Greenhough, T. J. \& Shrive, A. K. (2018). PLOS One 13, e0199175.

Keywords: collectin; surfactant protein D; bacterial recognition; innate immunity, pathogen-host interaction 